\title{
Clinical Observation of Self-Made Yin-Tonifying Ophiopogonis Decoction Combined with Rehabilitation New Liquid in the Treatment of Reflux Esophagitis with Deficiency of Stomach Yin
}

\author{
Liang Wang1, Li Liu' ${ }^{1}$ Zhenghui Liu ${ }^{2 *}$ \\ ${ }^{1}$ Shaanxi University of Chinese Medicine, Xianyang 712046, Shaanxi Province, China \\ ${ }^{2}$ The Affiliated Hospital of Shaanxi University of Chinese Medicine, Xianyang 712000, Shaanxi Province, China \\ *Corresponding author: Zhenghui Liu, lzh8462528@126.com
}

\begin{abstract}
Objective: To observe the efficacy of Yin-tonifying Ophiopogonis Decoction combined with Rehabilitation New Liquid in the treatment of reflux esophagitis (RE). Methods: Seventy-four patients with RE were included in the study and they were divided into an observation group and a control group using the random number table method with 37 cases in each group. The patients in the control group were given oral Yin-tonifying Ophiopogonis Decoction whereas the patients in the observation group were additionally treated with the Rehabilitation New Liquid. The clinical efficacy, traditional Chinese medicine (TCM) symptoms, and the efficiency of gastroscopy were compared between the two groups. Results: The total effective rate of clinical symptoms, TCM symptoms, and gastroscopy results between the observation group and the control group were significantly different $(\mathrm{P}<0.05)$ and were statistically significant. Conclusion: The clinical effect of Yin-tonifying Ophiopogonis Decoction combined with the Rehabilitation New Liquid in the treatment of RE is significant.
\end{abstract}

Keywords: Reflux esophagitis; Yin-tonifying Ophiopogonis Decoction; Rehabilitation New Liquid; Clinical observation

Publication date: July 2021; Online publication: July 30, 2021

\section{Introduction}

Reflux esophagitis (RE) refers to the reflux of gastric and duodenal contents into the esophagus, causing esophageal ulcers, mucosal inflammation, fibrosis, and other complications ${ }^{[1-2]}$. At present, gastrointestinal motility regulators, proton pump inhibitors, gastric mucosal protective agents, acid inhibitors, and other therapies are mostly used in clinical treatment, yet the curative effect is poor ${ }^{[3]}$. Liu Zhenghui who is a deputy chief physician designed the Yin-tonifying Ophiopogonis Decoction while aiming at the pathogenesis of RE with deficiency of stomach Yin which achieved remarkable curative effect in clinical application. In this study, the effect of Yin-tonifying Ophiopogonis Decoction combined with Rehabilitation New Liquid in the treatment of RE with deficiency of stomach Yin is discussed.

\section{Materials and methods}

\subsection{General information}

From July 2010 to July 2020, a total of 74 patients having reflux esophagitis with gastric Yin deficiency were selected and divided into a control group and an observation group using the random number table method with 37 cases in each group. There were 15 male patients and 12 female patients in the control group, age ranging from 18 to $65(45.27 \pm 16.088)$ years old, the course of disease ranging from 6 to 36 
(17.22 \pm 6.088$)$ months, and the endoscopic grading was A in 18 cases, B in 16 cases, and $\mathrm{C}$ in 3 cases. In the observation group, there were 14 male patients and 13 female patients, age ranging from 19 to 65 (44.65 $\pm 15.489)$ years old, the course of disease ranging from 8 to $41(18.43 \pm 7.708)$ months, and the endoscopic grading was $\mathrm{A}$ in 17 cases, $\mathrm{B}$ in 18 cases, and $\mathrm{C}$ in 2 cases. There were no significant differences in the general information between the two groups $(\mathrm{P}>0.05)$.

\subsection{Inclusion criteria}

The inclusion criteria were patients that had met the diagnostic criteria in the Diagnosis and Treatment of Reflux Esophagitis according to the Western medical diagnostic criteria ${ }^{[4]}$, patients that were diagnosed in accordance with the diagnostic criteria of medium-sized stomach yin deficiency syndrome in the Guiding Principles of Clinical Research of New Chinese Medicine (Trial) according to the traditional Chinese medicine diagnostic criteria, patients with age ranging from 18 to 65 years old, and those that had given informed consent to the research. According to the Los Angeles Classification (LA Classification) ${ }^{[5]}$, the esophageal mucosa was graded A, B, and C by endoscopy,

\subsection{Exclusion criteria}

Patients who had used anti-acid drugs or drugs affecting the gastrointestinal function within the past one month, patients that were allergic to the drugs used in this study, patients with a history of gastroesophageal surgery, malignant lesions, and peptic ulcer surgery, patients with heart, lung, liver, kidney, and other important organ dysfunctions, or women who were pregnant or lactating.

\subsection{Treatment methods}

The patients in the control group were given Yin-tonifying Ophiopogonis Decoction whereby the prescription was: Radix Ophiopogonis 12 g, Radix Pseudostellariae 15 g, Adenophora 12 g, Radix Rehmanniae Recens 10 g, Rhizoma Pinelliae Preparata 9 g, Caulis Bambusae in Taenia 10 g, stir-fried white peony $12 \mathrm{~g}$, Fructus Citri Sarcodactylis $10 \mathrm{~g}$, cuttlefish bone $20 \mathrm{~g}$, stir-fried bitter orange $12 \mathrm{~g}$, roasted grass $3 \mathrm{~g}$. One dose was given a day, decocted in $400 \mathrm{~mL}$ of water, administered warm before breakfast and dinner. The patients in the observation group were additionally given $10 \mathrm{~mL}$ of Rehabilitation New Liquid on the basis of the control group (Manufacturer: Sichuan Good Doctor Panxi Pharmaceutical Co., Ltd.; Approval number: SFDA approval number Z51021834). It was slowly administered orally, half an hour after meals, 3 times a day. Both groups were treated for 8 weeks.

\subsection{Evaluation}

The clinical efficacy, TCM symptoms, and the effective rate of gastroscopy after treatment were recorded. At the same time, the adverse conditions after treatment had been observed.

The clinical efficacy criteria: Recovery: The clinical symptoms disappeared, and gastroscopy was normal; Remarkable effect: The clinical symptoms significantly improved, local mucosa was slightly edematous under gastroscopy, but the area of congestion and erosion had reduced by more than 50\%; Efficient: The clinical symptoms were relieved and under gastroscopy, the local inflammation had improved; Inefficient: There was no relief of clinical symptoms and no change in the gastroscopy results.

The evaluation standard of TCM syndrome scores ${ }^{[6]}$ : Recovery: TCM syndrome score had decreased $\geq 95 \%$ and TCM symptoms disappeared; Remarkable effect: TCM syndrome score had decreased $\geq 70 \%$ but $<95 \%$ and TCM symptoms improved; Efficient: TCM syndrome score had decreased $\geq 30 \%$ but $<70 \%$ and TCM symptoms improved; Inefficient: TCM syndrome score had decreased $<30 \%$ and without improvement in TCM symptoms. 
The gastroscopy efficacy evaluation criteria were according to LA Classification: normal (0 point); Grade A (1 point); Grade B (2 point); Grade C (3 point). When the endoscopy score had reduced to 0 , the patient was considered cured, the decrease of 2 points was considered as marked effect, a decrease of 1 point was considered effective, and the endoscopy scores which did not change or increase were regarded as 1 point or inefficient. Total effective rate $(\%)=$ (cured cases + markedly effective cases + effective cases $)$ / total cases $\times 100 \%$.

\subsection{Statistical methods}

In this study, Statistical Package for the Social Sciences (SPSS) version 21.0 software was used for data processing. Counting data were represented as example $(\%)$ and chi-square $\left(\chi^{2}\right)$ test was used. $\mathrm{P}<0.05$ indicated statistically significant difference.

\section{Result}

\subsection{Comparison of clinical efficacy between the two groups}

The total effective rate of clinical symptoms in the observation group was higher than that in the control group, being statistically significant $(\mathrm{P}<0.05)$ (Table 1$)$.

Table 1. Comparison of clinical symptoms and efficacy between the two groups (n/\%)

\begin{tabular}{ccccccc}
\hline Group & $\begin{array}{c}\text { Number of } \\
\text { cases }\end{array}$ & Recovery & $\begin{array}{c}\text { Remarkable } \\
\text { effect }\end{array}$ & Efficient & Inefficient $\begin{array}{c}\text { Total } \\
\text { effective rate }\end{array}$ \\
\hline Observation group & 37 & $15(40.54 \%)$ & $11(29.73 \%)$ & $8(21.62 \%)$ & $3(8.11 \%)$ & $91.89 \% *$ \\
Control group & 37 & $13(35.14 \%)$ & $9(24.32 \%)$ & $5(13.51 \%)$ & $10(27.03 \%)$ & $72.97 \%$ \\
\hline
\end{tabular}

Note: Compared with the control group, $* \mathrm{P}<0.05$.

\subsection{Comparison of TCM syndrome efficacy between the two groups}

The total effective rate of TCM syndromes in the observation group was better than that in the control group, being statistically significant $(\mathrm{P}<0.05)$ (Table 2).

Table 2. Comparison of TCM syndrome efficacy between the two groups (n/\%)

\begin{tabular}{ccccccc}
\hline Group & $\begin{array}{c}\text { Number } \\
\text { of cases }\end{array}$ & Recovery & $\begin{array}{c}\text { Remarkable } \\
\text { effect }\end{array}$ & Efficient & Inefficient & $\begin{array}{c}\text { Total } \\
\text { effective rate }\end{array}$ \\
\hline Observation group & 37 & $16(43.23 \%)$ & $10(27.03 \%)$ & $7(18.92 \%)$ & $4(10.81 \%)$ & $89.19 \% *$ \\
Control group & 37 & $13(35.14 \%)$ & $9(24.32 \%)$ & $3(8.11 \%)$ & $12(32.43 \%)$ & $67.57 \%$ \\
\hline
\end{tabular}

Note: Compared with the control group, $* \mathrm{P}<0.05$.

\subsection{Comparison of gastroscopy efficacy between the two groups}

The total effective rate of esophageal mucosa treatment in the observation group under gastroscopy was better than that in the control group, being statistically significant $(\mathrm{P}<0.05)$ (Table 3). 
Table 3. Comparison of gastroscopy efficacy between the two groups before and after treatment (n/\%)

\begin{tabular}{ccccccc}
\hline Group & $\begin{array}{c}\text { Number } \\
\text { of cases }\end{array}$ & Recovery & $\begin{array}{c}\text { Remarkable } \\
\text { effect }\end{array}$ & Efficient & Inefficient & $\begin{array}{c}\text { Total effective } \\
\text { rate }\end{array}$ \\
\hline Observation group & 37 & $17(45.95 \%)$ & $13(35.14 \%)$ & $4(10.81 \%)$ & $3(18.11 \%)$ & $91.89 \% *$ \\
Control group & 37 & $12(32.43 \%)$ & $9(24.32 \%)$ & $7(18.92 \%)$ & $9(24.32 \%)$ & $75.68 \%$ \\
\hline
\end{tabular}

Note: Compared with the control group, $* \mathrm{P}<0.05$.

\section{Discussion}

$\mathrm{RE}$ is a common disease of the digestive system but with complex pathogenesis. It belongs to the categories of "swallowing acid," "nausea," "glomus and fullness," and "clamoring stomach" in traditional Chinese medicine. The deficiency syndrome of stomach Yin is one of the common clinical syndromes. The stomach prefers moisture and dislikes dryness which is regarded as harmony. If the deficiency of stomach Yin, the disharmony of qi, and the abnormal rise and fall are caused by exogenous factors of "six evils," unbalanced diet, or internal emotional injury, the flow of gastric contents is blocked, and they move into the esophagus along with the qi. Therefore, the treatment should be based on the principle of nourishing Yin and the stomach while lowering the inverse.

Yin-tonifying Ophiopogonis Decoction has been made by Liu ZhengHui, a deputy chief physician. It is composed of Radix Ophiopogonis, Radix Pseudostellariae, Adenophora, Radix Rehmanniae Recens, Rhizoma Pinelliae Preparata, Caulis Bambusae in Taenia, Fructus Citri Sarcodactylis, fried Radix Paeoniae Alba, stir-fried bitter orange, and roasted grass. The combination of all the above drugs has the effect of nourishing Yin, soothing the stomach, and lowering the reverse. Periplaneta americana belongs to the insect family, Blattellidae which has the efficacy of insect drugs to unclog collaterals, dissolve phlegm, remove blood stasis, and detoxify ${ }^{[7]}$. Rehabilitation New Liquid is an extract processed by modern technology from Periplaneta americana. In addition to nourishing blood vessels, Yin, and muscles, the Rehabilitation New Liquid can also strengthen and replenish deficiency. Modern pharmacological studies have proved that the Rehabilitation New Liquid has anti-inflammatory and anti-swelling effects. It can also promote the growth of new granulation tissue, accelerate the shedding of necrotic tissue, promote the repair of diseased tissues, and play a protective and repair role for the esophageal mucosa ${ }^{[8]}$.

The combination of Rehabilitation New Liquid and Yin-tonifying Ophiopogonis Decoction does not only enhance the effect of nourishing yin and benefiting the stomach, but it also improves the ability of anti-inflammation, protection, and repair of the esophageal mucosa. In this study, the clinical symptoms, TCM symptoms, and gastroscopy results of the two groups have been compared. The effective rate of the observation group was better than that of the control group, indicating that the effect of Yin-tonifying Ophiopogonis Decoction combined with Rehabilitation New Liquid in the treatment of RE was better than in administering Yin-tonifying Ophiopogonis Decoction alone. Rehabilitation New Liquid combined with Yin-tonifying Ophiopogonis Decoction does not only enhance the effect of nourishing yin and benefiting the stomach, but it also improves the ability of anti-inflammation, protection, and repair of the esophageal mucosa.

\section{Disclosure statement}

The authors declare that there is no conflict of interest. 


\section{References}

[1] Yu Y, Song S, Zhou X, 2018, Advances in Pathogenesis and Diagnosis and Treatment of Reflux Esophagitis. Journal of Pediatrics, 40(5): 490-4.

[2] Liu X, Fu Q, 2017, Clinical Efficacy of Omeprazole Combined with Trimebutine in the Treatment of Reflux Esophagitis. Journal of Tropical Medicine, 17(12): 1656-8.

[3] Wei Q, Wei M, Wei Y, et al., 2020, Clinical Research of Treating Reflux Esophagitis with Platycodon Grandiflorum and Fructus Aurantii Decoction Combined with Western Medicine. Journal of Shandong University of Traditional Chinese Medicine, 44(05): 550-4.

[4] Lu X, Zhang T, 2004, Guidelines for the Diagnosis and Treatment of Reflux Esophagitis. Chinese Journal Of Digestive Endoscopy, 21(4): 221-2.

[5] Lundell LR, Dent J, Bennett JR, et al., 1999, Endoscopic Assessment of Esophagitis: Clinical and Functional Correlates and Further Validation of the Los Angeles Classification. Gut, 45:172-80.

[6] Zheng X, 2002, Clinical Research Guidelines of New Chinese Medicine (Trial), China Medical Science and Technology Press, Beijing, 341-3.

[7] Hao X, Li N, Bai H, et al., 2020, Li Diangui's Experience in Treating Chronic Atrophic Gastritis with Intestinal Metaplasia. Chinese Journal of Traditional Chinese Medicine and Pharmacy, 35(03): 12369 .

[8] Zhang D, Zhang Y, Yang D, 2019, Clinical Study of Rehabilitation New Liquid Combined with Pantoprazole in the Treatment of Reflux Esophagitis. Modern Drugs and Clinics, 34(03): 686-9. 